\title{
Problèmes posés par l'étude histophysiologique quantitative de quelques glandes endocrines chez les Téléostéens
}

\author{
Madeleine Olivereau \\ Laboratoire de Physiologie de l'Institut Océanographique \\ Paris 5, France
}

\begin{abstract}
Problems of quantitative histophysiological research of some endocrine glands in teleosts. The need to combine qualitative description and quantitative data is stressed and the interest in comparing them is emphasized in histophysiological studies of teleost endocrines. The pituitary gland has a rather complex structure; the numeration of the various cell types is slow, tedious, and not very informative. Cytological and histochemical processes are more often used to examine histological modifications after experimental treatments or during fish migration. Since thyroid gland, interrenal and islets of Langerhans are not individualized in teleosts, their histophysiological study is more difficult than in mammals, and their weight changes are impossible to evaluate. Caryometric determinations were performed, as well as measurements of epithelial cell height of the thyroid gland, and autohistoradiographic studies were made. The determination of the number of follicles shows that salmon thyroid is larger than trout thyroid, and this difference in size may partly explain why salmon is more difficult to radiothyroidectomize than trout. Planimetric and caryometric studies and numeration of cell nuclei belonging to a constant area of interrenal tissue were undertaken. The quantitative data were submitted to statistical evaluation and their significance was established. These criteria demonstrate that interrenal is strongly stimulated after a treatment with metopiron, aldacton or reserpin; conversely, hypophysectomy or cortisol administration reduces this activity. A transfer into distilled water induces only a slight stimulation. The transformation of a sedentary parr into a migrating smolt (Salmo salar) is accompanied by a hypertrophy of adrenals, temporarily reinforced after a transfer into seawater. Corpuscles of STANnius and endocrine pancreas were also submitted to planimetric or caryometric studies and results are briefly discussed.
\end{abstract}

\section{INTRODUCTION}

Il y a quelques décennies, l'histophysiologie des glandes endocrines était essentiellement basée sur la description qualitative de leurs modifications structurales, mais celle-ci risquait parfois d'être assez subjective. Ces descriptions tendent de plus en plus à être complétées par l'emploi de techniques dont les résultats peuvent être exprimés quantitativement et discutés plus objectivement par l'application des calculs statistiques.

Cette nécessité de l'introduction de données quantitatives en histophysiologie 
endocrinienne était particulièrement impérieuse chez les Téléostéens dont plusieurs glandes - thyroïde, système corticosurrénalien, pancréas Langerhansien - sont diffuses; elles sont donc impossibles à prélever à l'état pur et en totalité, et ne permettent pas l'obtention de données pondérales au cours du cycle biologique de ces animaux ou en réponse à différents traitements expérimentaux. Mais chaque glande pose un problème particulier et demande un procédé adapté à sa structure anatomique ou histologique. Le test le plus souvent adopté est celui de la caryométrie ou mesure du diamètre ou du volume nucléaire, applicable à n'importe quel tissu, mais nous verrons qu'il ne suffit pas toujours à exprimer l'activité d'un organe.

Nous envisagerons successivement le cas de l'hypophyse, de la thyroïde, du système hypophyso-corticosurrénalien et du pancréas endocrine chez plusieurs Téléostéens, en décrivant les techniques mises au point, mentionnant quelques-unes des valeurs obtenues, mais en présentant conjointement des images histologiques afin de mieux apprécier les activités des organes étudiés.

\section{HYPOPHYSE}

Bien qu'individualisée, l'hypophyse des Téléostéens n'a presque jamais été étudiée pondéralement, car toute pesée d'un organe frais retentit fâcheusement sur sa structure histologique, surtout sur l'adénohypophyse très fragile. De plus, l'importance physiologique du tractus hypothalamo-hypophysaire impose maintenant l'étude de l'hypophyse avec une tige intacte la rattachant encore à l'hypothalamus. Le rapport du poids hypophysaire au poids corporel augmente pendant la reproduction chez le Saumon (FONTAINE et al. 1950), la femelle ayant une hypophyse plus lourde que celle du mâle (Olviereau 1954); chez l'Anguille femelle traitée par un extrait thyréostimulant, le poids hypophysaire s'accroît (FoNTAINE 1961) en liaison probable avec un développement massif de cellules gonadotropes mis en évidence chez le mâle (OlIvereau 1961a).

L'adénohypophyse des Téléostéens, comme celle de la plupart des Vertébrés, est un organe particulièrement complexe, comprenant 5 ou 6 types cellulaires chromophiles dans la pars distalis et 1 ou 2 dans la pars intermedia. La caryométrie offre l'avantage d'être utilisable pour toutes les catégories de cellules. Toute hypertrophie nucléaire est généralement associée à un accroissement de la taille du nucléole et au développement de l'ergastoplasme, indiquant une stimulation cellulaire; celle-ci est vérifiable, soit par des dosages chimiques ou biologiques de la substance élaborée, soit par l'étude des modifications enregistrées au niveau des organes récepteurs.

Les dimensions cellulaires fournissent également un critère d'activité valable; la raréfaction des granulations cytoplasmiques dans une cellule hypertrophiée laisse supposer un turnover accéléré du produit de sécrétion: la synthèse est importante, mais l'excrétion paraît supérieure et l'on assiste à une disparition progressive de la réserve hormonale, tandis que le taux de l'hormone crô̂t dans le milieu intérieur. Enfin, la présence d'une activité mitotique confirme un diagnostic de stimulation glandulaire. Inversement, une cellule peu active, dont la sécrétion ou l'excrétion est expérimentalement inhibée, est de taille réduite et souvent riche en granulations. Notons que si ces granulations ne représentent généralement pas l'hormone active, elles en constituent 

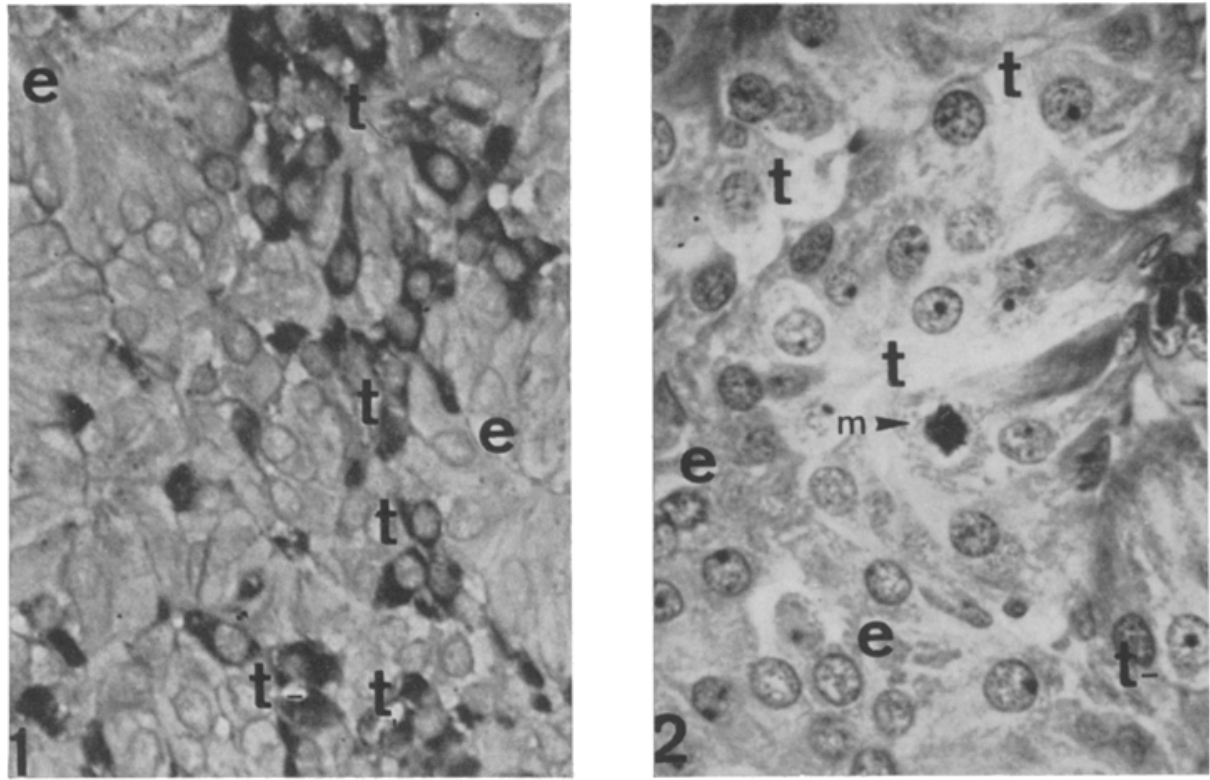

Fig. 1: La pars distalis rostrale de l'hypophyse de l'Anguille contient des cellules érythrosinophiles (e), disposées en follicules, sécrétant une hormone de type prolactinique, et des cellules thyréotropes $(t)$, renfermant des glycoprotéides, intensément colorées par l'aldéhyde fuchsine. $\mathrm{X} 800$ fois

Fig. 2: Chez l'Anguille dont la majeure partie de la thyrö̈de a été détruite par l'injection de ${ }^{131}$ I $(450 \mu \mathrm{c}$, poids $32,5 \mathrm{~g})$, les cellules thyréotropes $(\mathrm{t})$ présentent déjà une vacuolisation, un mois après le début du traitement; les granulations glycoprotidiques ont disparu. Les noyaux et les nucléoles sont hypertrophiés, une mitose (m) est visible. Les cellules érythrosinophiles (e) sont moins fortement stimulées. X 800 fois

souvent le support et sont en relation étroite avec la substance élaborée. Si l'inhibition fonctionnelle est très prolongée, l'atrophie cellulaire s'accentue et peut s'accompagner d'une disparition de la charge granulaire. C'est le cas, par exemple, des cellules thyréotropes de l'Anguille soumise à un traitement thyroxinien pendant plus d'un mois (Olivereau 1960a).

En pratique, ces principaux critères d'activité (caryométrie, dimensions cellulaires) n'ont pas été sérieusement quantifiés chez les Téléostéens, en partie à cause de l'extrême variabilité de forme des cellules selon le plan de la coupe. Ce n'est qu'incidement que nous les avons utilisés pour évaluer, par exemple, la réponse des cellules thy réotropes de l'Anguille à une radiothyroïdectomie (Olivereau 1963a) ou celle des éléments corticotropes après un traitement par la métopirone (OLIVEREAU 1965) ou l'aldactone (Otrvereau 1964). Ajoutons que cette étude gagne à être réalisée en fonction du temps afin de mieux apprécier l'évolution des modifications de la cellule selon la durée du traitement.

Considérant la multiplicité des types cellulaires de la pars distalis, il paraissait fructueux d'évaluer le pourcentage de chaque catégorie d'éléments. Cette technique fut très utilisée il y a quelques dizaines d'années, mais elle est fastidieuse, imposant 

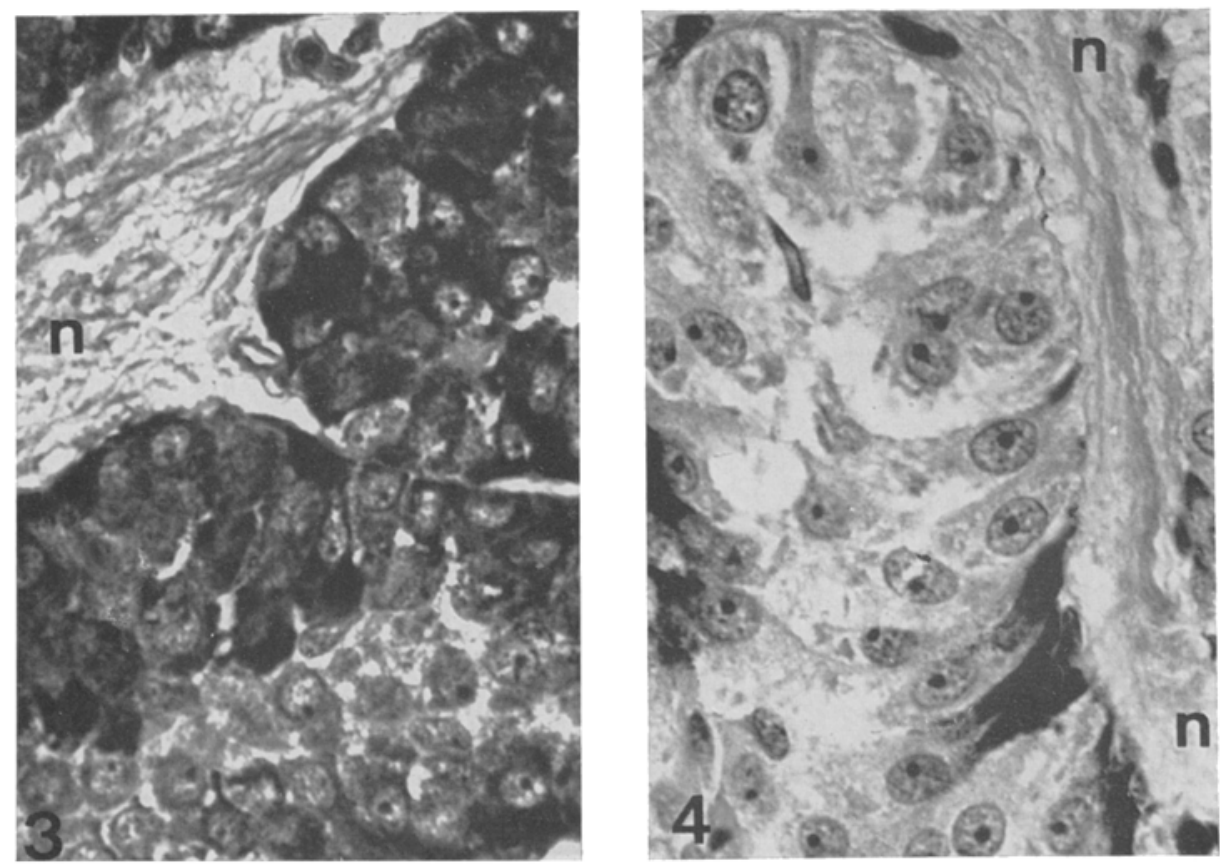

Fig. 3: A la limite de la neurohypophyse (n), les cordons de la pars distalis rostrale contiennent une couche de cellules corticotropes fortement granulées, colorables par l'hématoxyline au plomb de Mac Conaill chez l'Anguille. X 1.000 fois

Fig. 4: Un traitement par la métopirone pendant 21 jours entraîne chez l'Anguille une dégranulation rapide et totale des cellules corticotropes dont plusieurs sont vacuolisées. L’hypertrophie nucléaire et nucléolaire est tout à fait évidente. X 1.000 fois

pour une mesure précise le dessin des champs explorés, puis le dénombrement de milliers de cellules, en particulier chez le Saumon de l'Atlantique, Salmo salar L. au cours de ses migrations et de la reproduction (Olivereau 1954). Or, jusqu'ici les numérations ont été faites en ne distinguant que trois types cellulaires: cellules acidophiles, basophiles et chromophobes. Mais le taux relatif d'une classe d'éléments dépend essentiellement des variations simultanées des autres classes. Le résultat final apporte donc peu d'information; nous avons renoncé à cette technique longue et fatigante, et nous ne reproduirons pas ici tous les tableaux et les courbes résumant les valeurs obtenues antérieurement (OLIVEREAU 1954, p. 152-156).

Ce procédé, bien que presque abandonné, est encore utilisé sur des espèces de taille réduite, ce qui limite le nombre des cellules à étudier (exemple chez Xiphophorus, Schreibman 1964). Grâce aux techniques histologiques et histochimiques et à une analyse expérimentale variée (BENOIT \& DA LAGE 1963), on sait maintenant que les cellules acidophiles ou séreuses comprennent les éléments alpha sécrétant l'hormone somatotrope, epsilon élaborant l'hormone corticotrope, et eta produisant une hormone de type prolactinique, et que les basophiles, mucoïdes ou glycoprotéiques englobent les éléments gonadotropes beta et gamma et les thyréotropes delta; on peut donc 

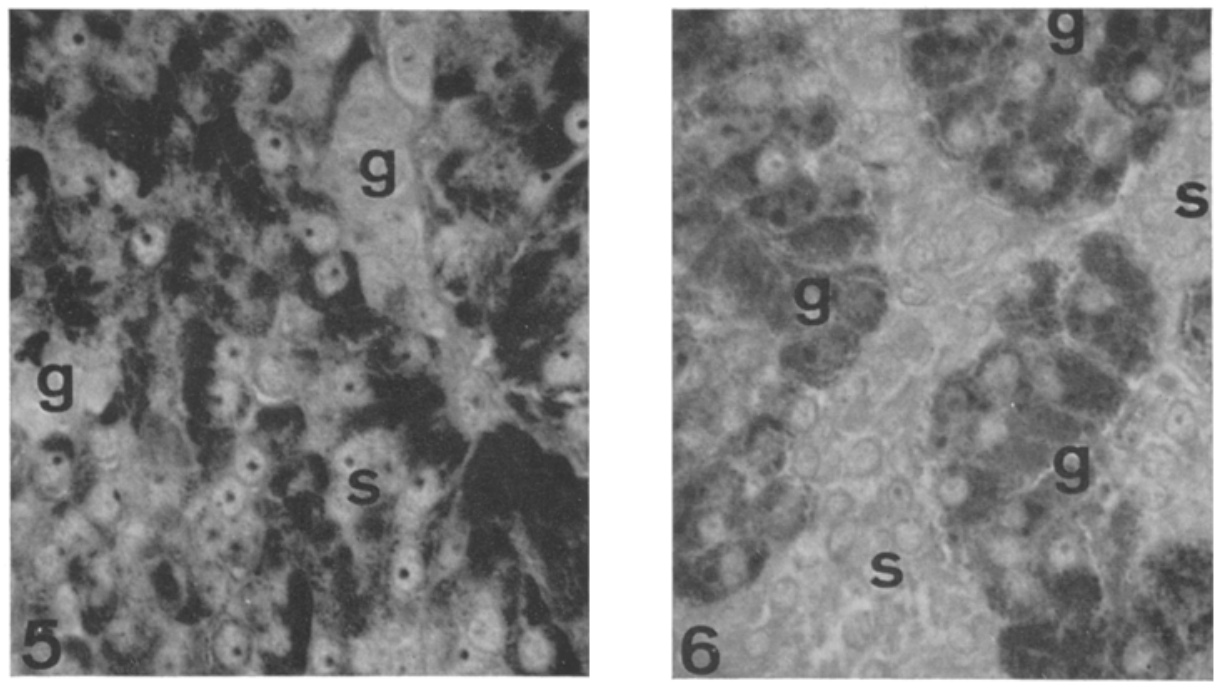

Fig. 5: Dans la pars distalis proximale de l'Anguille immature, on note la présence de nombreuses cellules somatotropes (s) ou alpha, vivement colorées par l'Azan, qui apparaissent ici en noir grâce au filtre vert utilisé pour la photographie. Les cellules gonadotropes ( $\mathrm{g}$ ) sont très peu nombreuses et à peine différenciées. Leur noyau est petit, le nucléole peu visible. Elles ne renferment que des traces de glycoprotéides. X 800 fois

Fig. 6: Les cellules somatotropes (s) sont moins nombreuses et apparaissent moins actives chez les Anguilles mâles dont la maturation génitale a été induite, soit par l'injection de prolans, en eau de mer, soit simplement par l'injection d'hormone thyréotrope (probablement contaminée par des traces de facteur lutéinisant), en eau douce. Par contre, les cellules gonadotropes (g) prédominent. Elles sont globuleuses, avec de grosses granulations glycoprotidiques très colorables par l'aldéhyde fuchsine. X 800 fois

s'interroger sur la signification réelle de numérations basées sur l'identification de deux classes de cellules chromophiles.

En réalité, l'étude cytophysiologique de l'hypophyse reste surtout qualitative et histochimique, et il n'est pas besoin de numérations pour être sûr que la radiothyroïdectomie, chez l'Anguille par exemple, s'accompagne d'une hypertrophie marquée des cellules thyréotropes qui se dégranulent et se vacuolisent, d'une augmentation du volume nucléaire et nucléclaire, ainsi que le montre la comparaison des figures 1 et 2 . Il en est de même chez le Cyprin (Olrvereau 1962) ou la Truite radiothyroïdectomisés (Olivereau, la Roche \& Woodall 1964).

Les cellules corticotropes (figure 3) réagissent aussi très fortement à un traitement par la métopirone (figure 4) ou l'aldactone, en réponse au blocage de la synthèse des corticostéroïdes ou à l'inhibition de l'utilisation périphérique de certains d'entre eux.

Voici un dernier exemple: la figure 5 représente les cellules somatotropes très développées chez l'Anguille immature dont la biologie est orientée vers une croissance lente, mais poursuivie pendant plusieurs années; les cellules gonadotropes sont à peine visibles et presque indifférenciées. La figure 6 correspond à une Anguille mâle en pleine reproduction: les cellules gonadotropes sont globuleuses avec de grosses granulations 
glycoprotidiques; elles prédominent très largement et nulle numération n'est nécessaire pour affirmer le virage physiologique qui s'est manifesté, l'activité somatotrope ayant fortement régréssé au profit d'une activité gonadotrope dominante.

Récemment, la localisation de quelques isotopes a été précisée par autohistoradiographie dans l'hypophyse du Cyprin (DEMnNatri) et du Muge (LERAY), ces résultats ayant été discutés antérieurement (Olivereau 1963b). Bien que les données obtenues soient encore d'ordre qualitatif, c'est une technique qui se révèle intéressante et susceptible d'une quantification ultérieure.

La microscopie électronique se prête-t-elle mieux à une étude quantitative? Jusqu'ici, celle-ci a été limitée à la mensuration des dimensions des granules cytoplasmiques. Mais ces valeurs ne permettent pas toujours d'identifier avec certitude la nature de la cellule examinée et selon les états physiologiques la taille des granules présente d'amples variations (Follenius 1963).

L'histophysiologie adénohypophysaire est donc encore essentiellement abordée par des techniques cytologiques ou histochimiques, à la suite de traitements expérimentaux variés, dont les résultats apparaissent démonstratifs et l'interprétation relativement aisée. Mais ces données n'ont été que peu soumises jusqu'ici à une analyse quantitative difficile, longue et parfois peu informative.

\section{THYROÏDE}

Anatomiquement, il n'existe pas une glande thyroïde individualisée chez la plupart des Téléostéens, mais des nodules plus ou moins compacts de follicules thyrö̈diens, dispersés dans la région branchiale, à proximité des veines jugulaires et de l'artère aorte, ou même autour du coeur dans le cas de l'Uegitglanis zammaranoi Gianferrari (Oltvereau 1960b) ou dans le rein céphalique (Cyprin, Xiphophorus, Typhlogarra... revue in Olivereau 1960c).

La caryométrie dans les cellules de l'épithélium est en fait assez peu utilisée pour les acini des Téléostéens: quand la thyrö̈de est peu active, le noyau est de très petite taille, souvent de forme irrégulière, et son volume ne peut être déterminéavec précision.

La mesure la plus fréquemment réalisée est celle de la hauteur épithéliale qui varie en fonction de l'état d'activité de la glande, mais les variations selon les follicules et selon les régions d'une même vésicule peuvent être importantes, surtout dans les zones périphériques qui paraissent bénéficier d'une vascularisation moindre. Cette mesure demande à être complétée par l'examen de la colloïde, la recherche d'une activité mitotique et le développement de l'ergastoplasme, comme dans le cas d'un traitement par la métopirone (Olivereau 1965) ou la prolactine ovine (Olivereau 1966) (Tableau 1).

L'autohistoradiographie, après injection d'iode radioactif ${ }^{131} \mathrm{~J}$ ou ${ }^{125} \mathrm{~J}$, permet aussi d'évaluer la liaison organique de l'isotope dans les vésicules: l'intensité du noircissement de l'émulsion photographique à fine résolution qui recouvre la coupe est proportionnelle à la quantité d'iode lié. Ce noircissement peut être apprécié par densitométrie, mais la précision de ces mesures n'atteint cependant pas celle fournie 

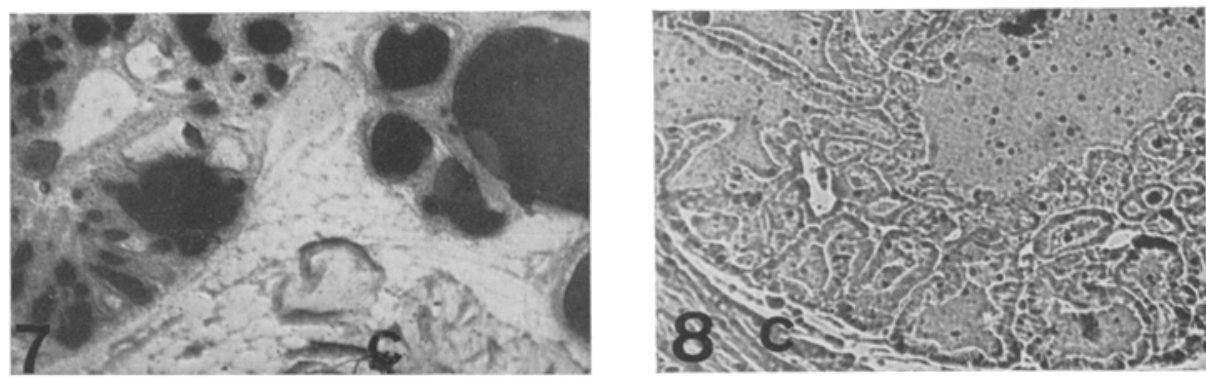

Fig. 7: Autohistoradiographie de la glande thyroïde d'un Congre témoin. L'épithélium est visible après coloration par l'hémalun, à travers l'émulsion photographique. La collö̈de contient des quantités importantes de ${ }^{131} \mathrm{~J}$ lié organiquement. Le conjonctif (c) ne fournit pas d'autoradiographie positive. Sacrifice 4 jours après l'injection de ${ }^{131} \mathrm{~J}$. X 225 fois

Fig. 8: Autohistoradiographie d'un Congre traité pendant 12 jours par le thiouracile qui bloque la synthèse de l'iode hormonal. L'épithélium reste visible sous l'émulsion. La colloìde ne contient plus d'iode marqué lié organiquement. Les taches noires sont liées à une diffraction de la lumière produite par l'émulsion. X 225 fois

par les dosages biochimiques et la radiochromatographie. Les figures 7 et 8 permettent de comparer l'autohistoradiographie foumie par la thyroïde d'un Congre (Conger conger L.) témoin et celle provenant d'un animal traité par le thiouracile qui inhibe la liaison organique de l'iode dans la glande.

De même, l'autohistoradiographie met en évidence une hyperactivité thyroïdienne chez le Saumon pendant la smoltification, en accord avec les résultats de l'étude biochimique et histologique (FonTArne et al. 1952).

\section{Tableau 1}

Mesure de la hauteur de l'épithélium thyroïdien chez l'Anguille

On voit ainsi que la métopirone et la prolactine n'ont plus d'effet chez l'Anguille hypophysectomisée, mais stimulent significativement la thyroide de l'animal intact

\begin{tabular}{|lccc|}
\hline Traitement & $\begin{array}{c}\text { Nombre } \\
\text { d'animatix }\end{array}$ & $\begin{array}{c}\text { Hauteur } \\
\text { épithéliale } \\
\text { en } \mu\end{array}$ & $\begin{array}{c}\text { Signifi- } \\
\text { cation }\end{array}$ \\
\hline Témoins non injectés & 14 & $8,45 \pm 0,37$ & \\
Témoins injectés de NaCl & 8 & $8,85 \pm 0,84$ & $\mathrm{P}<0,01$ \\
Métopirone & 33 & $10,50 \pm 0,37$ & $\mathrm{P}<0,001$ \\
Hypophysectomisés & 7 & $4,30 \pm 0,50$ & $\mathrm{P}=0,10$ \\
Hypophysectom. + Métopirone & 10 & $5,52 \pm 0,46$ & $\mathrm{P}<0,001$ \\
Témoins injectés de NaCl & 19 & $6,15 \pm 0,19$ & $\mathrm{P}<0,001$ \\
Prolactine & 28 & $12,47 \pm 0,41$ & \\
Hypophysectomisés & 13 & $4,46 \pm 0,20$ & \\
Hypophysectom. + Prolactine & 19 & $4,59 \pm 0,18$ & \\
\hline
\end{tabular}

La glande thyroïde de presque tous les Téléostéens étant diffuse, son ablation chirurgicale est irréalisable, mais sa destruction par l'iode radioactif est possible, à condition d'injecter plusieurs doses, car les fixations de ${ }^{131} \mathrm{~J}$ sont généralement faibles 
chez les Poecilothermes. Il importe ensuite de vérifier la totalité de la radiothyroïdectomie par un examen histologique de coupes sériées de la région thyroidienne (OrIVEREAU 1957). Or, nous avons été surprise de constater que chez des individus de poids et taille identiques, maintenus dans des conditions identiques, l'injection de doses égales d'iode radioactif (100 $\mu \mathrm{c}$ de ${ }^{131} \mathrm{~J}$ par mois, pendant 6 mois) provoque une thyroïdectomie sensiblement totale chez la Truite (Salmo gairdneri) (Olvereau et al. 1964) alors que chez le Saumon du Pacifique (Oncorbynchus tsharwytscha) celle-ci n'est que partielle: il reste environ 10 à $15 \%$ des follicules initialement présents, plusieurs d'entre eux étant d'ailleurs d'aspect anormal. Il paraissait difficile d'attribuer ces réponses divergentes à des différences de radiosensibilité du tissu thyroïdien chez deux espèces aussi voisines. Mais nous avons fait une simple numération des vésicules thyroïdiennes dans la région médiane du nodule antérieur et constaté chez la Truite la présence d'environ 150 follicules par coupe alors qu'il y en a plus de 300 chez le Saumon. La teneur en iode stable et la fixation de ${ }^{131} \mathrm{~J}$ légèrement supérieures chez le Saumon (LA RocHE et al. 1965) s'expliquent vraisemblablement par un volume glandulaire supérieur. Ia quantité relative d'isotope disponible pour chaque follicule serait donc inférieure chez le Saumon, par rapport à la Truite, fait qui pourrait être en partie responsable de la radiothyroïdectomie subtotale chez cette espèce. La réaction des cellules thyréotropes hypophysaires du Saumon est d'ailleurs aussi incomplète, quelques granulations glycoprotidiques persistant dans le cytoplasme (Olrvereau \& LA Roche 1965).

\section{INTERRÉNAL}

L'interrénal ou homologue fonctionnel du tissu corticosurrénalien des Vertébrés supérieurs (Leloup-HATEY 1964a) est situé selon les descriptions de GIACOMINI (1908a et b) dans les parois des veines cardinales postérieures et des veines cardinales antérieures primitives ou jugulaires chez l'Anguille. Nous avons vérifé cette localisation et constaté que l'interrénal se retrouve sur une longueur de 11 à 13 millimètres chez des mâles pesant une soixantaine de grammes. On observe un petit nodule antérieur constitué par du tissu presque pur, puis un manchon périveineux dont les derniers îlots ne sont plus dans la paroi même de la veine, mais se développent dans le tissu lymphoïde qui les entoure complètement. Cette vaste répartition des nodules de l'interrénal ne rend donc pas sa pesée ni son ablation chirurgicale aisées, bien que celles-ci aient été tentées.

Une expérimentation variée (hypophysectomie, injection d'hormone corticotrope ou ACTH...) a permis de constater des variations pondérales importantes de «la glande» prélevée dans la veine cardinale antérieure (LELOUP-HATEY 1964a). L'ablation des veines cardinales postérieures contenant de l'interrénal a été réalisée (CHESTER JONEs et al. 1964) et la répercussion de l'interrénalectomie sur le métabolisme électrolytique précisée (Chester Jones et al. 1965). Seule l'étude planimétrique des dessins des aires correspondantes à chaque zone glandulaire, faits à partir de coupes sériées, permet de connaître la répartition réelle de l'interrénal par rapport au sinus de Cuvier où aboutissent les veines cardinales antérieures et postérieures. Les valeurs obtenues 
indiquent que la veine cardinale antérieure contient seulement 35 à $40 \%$ du tissu interrénalien total. Bien que variant parallèlement au volume de toute la glande et reflétant celui-ci, les données pondérales indiquées ne représentent donc pas «la glande», mais seulement un peu plus du tiers de celle-ci.

De même, la planimétrie montre que les interrénalectomies pratiquées entrânent sûrement un état marqué d'hypocorticisme responsable des modifications électrolytiques observées, mais il reste environ le tiers du tissu qui subit très probablement une forte hypertrophie compensatrice.

L'évaluation du volume glandulaire par planimétrie de coupes sériées est utile également chez la Truite: la petitesse et la dissémination des îlots d'interrénal dans le tissu lymphoïde du rein céphalique font volontiers croire que ce tissu est peu développé. Il n'en est rien, les valeurs trouvées étant voisines de celles du jeune Saumon sédentaire ou parr.

Chez le Saumon, la transformation du parr sédentaire en smolt migrant vers l'Océan Atlantique s'accompagne d'une activation de l'interrénal dont les aspects qualitatifs ont été décrits antérieurement (Fontaine \& Olrvereau 1957, Olivereau 1960d). La planimétrie permet de préciser l'hypertrophie de ce tissu (FonTAINE $\& x$ Olivereau 1959). Ainsi, chez le parr mâle, le volume de l'interrénal évalué après fixation est en moyenne de $1,35 \mathrm{~mm}^{3}$ pour 100 grammes de poids corporel; il s'élève à 2,14 $\mathrm{mm}^{3}$ chez le pseudo-smolt mâle (animal ayant subi une smoltification plus ou moins complète en captivité) et à $2,54 \mathrm{~mm}^{3}$ chez le smolt mâle et $2,19 \mathrm{~mm}^{3}$ dez le smolt femelle. En d'autres termes, en plus des modifications qualitatives montrant une stimulation de l'interrénal pendant la smoltification, on voit que le volume de ce tissu est presque doublé. Si les smolts sont placés en eau de mer, l'interrénal est à nouveau temporairement stimulé, et la planimétrie montre que le volume varie de 2 à $4,5 \mathrm{~mm}^{3}$ pour $100 \mathrm{~g}$ selon les animaux, avec une valeur moyenne de $2,7 \mathrm{~mm}^{3}$. Après 14 jours en eau de mer, le volume redevient à peu près similaire à celui observé en eau douce. Ces valeurs sont résumées dans le tableau 2.

Chez l'Epinoche (Gasterosteus aculeatus) STANwORTH (1953) recommande de faire la planimétrie sur une seule coupe représentative de l'ensemble de l'interrénal pour apprécier son activité. La solution de cette «coupe représentative» nous paraît bien subjective et nous avons souligné antérieurement (1960d) que si cette technique peut à la rigueur convenir pour des espèces de petite taille, elle n'est nullement applicable au Saumon, l'abondance de l'interrénal variant considérablement d'un niveau à l'autre. Seule la planimétrie de l'ensemble du tissu peut refléter fidèlement son activité.

Les variations dù volume glandulaire ne sont pas les seules intéressantes à considérer, celles du volume nucléaire et des dimensions cellulaires apportent aussi des informations utiles. C'est ainsi que nous avons été amenée à dénombrer les noyaux sur une unité de surface déterminée et constante $\left(1.600 \mu^{2}\right)$ et à établir une densité nucléaire qui est d'autant plus faible que les cellules sont hypertrophiées. Inversement, une densité élevée de noyaux correspond à des cellules atrophiées. Parallèlement, la mesure des diamètres nucléaires vient confirmer l'hypertrophie ou l'atrophie des noyaux selon l'état physiologique des animaux ou les conditions expérimentales. 
Tableau 2

Mesure planimétrique de l'interrénal du Saumon pendant la smoltification

\begin{tabular}{|c|c|c|c|}
\hline Traitement ou stade & $\begin{array}{l}\text { Nombre } \\
\text { d'animaux }\end{array}$ & $\begin{array}{l}\text { Volume d'interrénal } \\
\text { par gramme d'animal } \\
\text { en } \mathrm{mm}^{3} \times 10^{5}\end{array}$ & Signification \\
\hline Parrs mâles & 4 & $1.344,7 \pm 24,3$ & \\
\hline Pseudo-smolts mâles & 5 & $2.137,8 \pm 215,7$ & \\
\hline Smolts mâles & 6 & $2.540,3 \pm 371,5$ & $P<0,01$ \\
\hline Smolts femelles & 8 & $2.187,8 \pm 256$ & $P<0,01$ \\
\hline $\begin{array}{l}\text { Smolts ô et } q, 3 \mathrm{~h} \text {. ̀े } 14 \mathrm{j} \text {. en } \\
\text { eau douce }\end{array}$ & 11 & $1.982 \pm 138$ & \\
\hline $\begin{array}{l}\text { Smolts } \delta \text { et } q, 30 \mathrm{~min} \text {. } 3 \mathrm{~h} \text {. en } \\
\text { eau de mer }\end{array}$ & 4 & $3.015 \pm 508$ & $P<0,01$ \\
\hline $\begin{array}{l}\text { Smolts ô et } q, 1 \text { à } 14 \mathrm{j} \text {. en } \\
\text { eau de mer }\end{array}$ & 12 & $2.708 \pm 205$ & $0,01<\mathrm{P}<0,02$ \\
\hline
\end{tabular}

Ces techniques avaient été initialement appliquées à l'Anguille argentée femelle, avec Fromentin (1954), les résultats montrant après hypophysectomie une atrophie de la surface cellulaire voisine de $30 \%$ et une atrophie nucléaire un peu moins marquée.

Depuis, nous avons largement utilisé ces deux techniques dont les résultats sont toujours soumis au calcul statistique. Divers traitements expérimentaux - métopirone, aldactone, réserpine - provoquent une stimulation marquée de l'interrénal, bien que temporaire dans le cas de la réserpine (Olivereau 1963c), traduite par une forte hypertrophie cellulaire et nucléaire, souvent confirmée par la présence de mitoses. La comparaison de la figure 9 concernant une Anguille hypophysectomisée et de la figure 10 illustrant l'effet d'un traitement par l'aldactone montre bien l'hyperactivité induite par cette substance perturbant l'équilibre électroly tique de l'animal (OLrvereau \& Chartier 1965). L'hypertrophie du nucléole est également caractéristique, mais jusqu'ici elle n'a jamais été chiffrée chez les Téléostéens.

Nous ne pouvons envisager dans cette communication les modifications qualitatives simultanément observées au cours de ces traitements expérimentaux; signalons que d'autres processus provoquent une hypertrophie cellulaire sans retentir nettement sur le volume nucléaire, suggérant l'idée d'une stimulation moins importante que dans les expériences précédentes. Enfin, d'autres traitements déterminent une atrophie cellulaire considérable, comme l'hypophysectomie (figure 9) ou l'administration de cortisol ( $1 \mathrm{mg}$ par jour pour des Anguilles pesant de 55 à 75 grammes). Notons toutefois que si l'involution cellulaire est du même ordre de grandeur dans les deux cas, l'effet de l'hypophysectomie entraîne une régression nucléaire beaucoup plus marquée qu'une surcharge de l'organisme en cortisol. L'ensemble des valeurs obtenues au cours de cette expérimentation se trouve résumé dans le tableau 3.

Il faut noter que ces techniques doivent être appliquées à des coupes relativement fines afin que les numérations conservent une certaine précision: il est évident qu'un diamètre nucléaire de 4 ou $5 \mu$ doit être mesuré à un fort grossissement au moins égal à 1.500 fois et sur une coupe dont l'épaisseur ne dépasse pas $5 \mu$, sous peine de faire des mesures dépourvues de précision. 

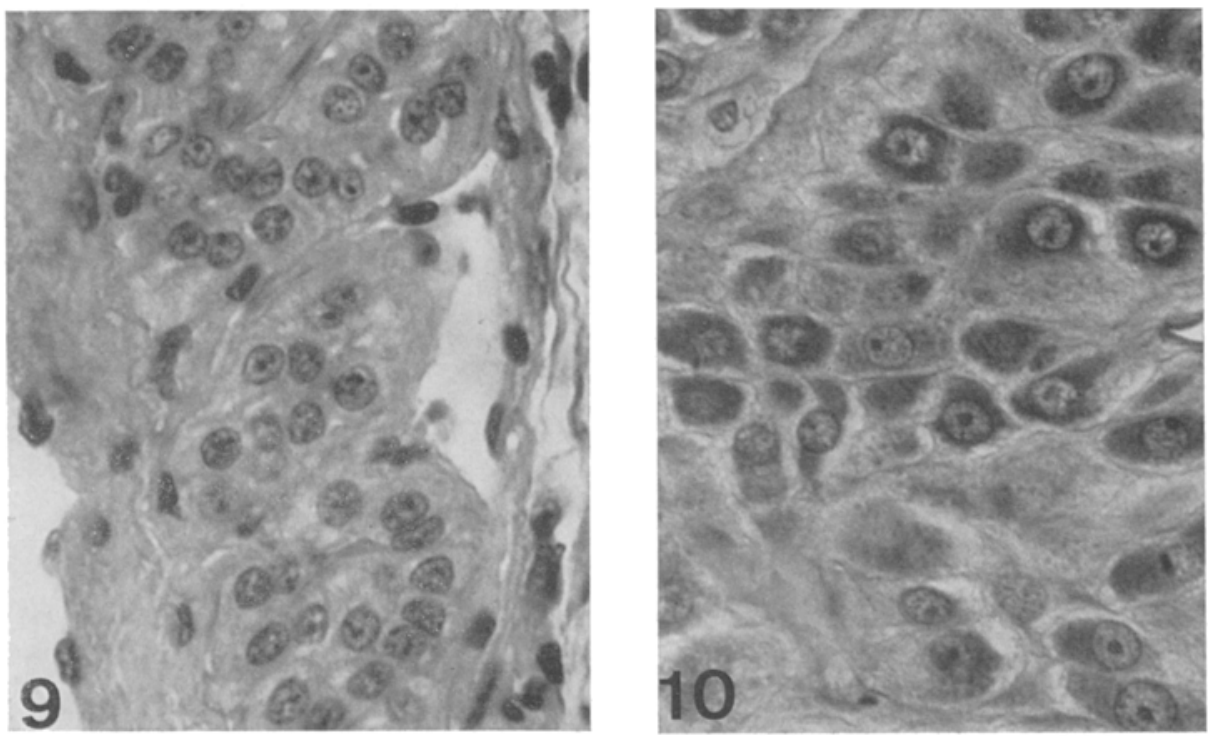

Fig. 9: Interrénal d'une Anguille hypophysectomisée depuis 15 jours. Les noyaux sont de forme irrégulière, les nucléoles peu visibles. Les cellules aux limites peu discernables sont atrophiées. X 1.100 fois

Fig. 10: Interrénal d'une Anguille intacte ayant reçu 9 injections (25 $\mathrm{mg}$ tous les 2 jours) d'aldactone. Les cellules aux limites nettes sont hypertrophiées ainsi que les noyaux et les nucléoles. La zone périnucléaire est riche en granulations fortement éosinophiles, mais aussi colorables par le bleu d'alizarine. X 950 fois

Van Overbeeke (1960) a abordé ce problème quantitatif d'une façon similaire à la nôtre. Par planimétrie du dessin d'îlots cellulaires, l'auteur calcule la surface moyenne d'une cellule et constate qu'un traitement par l'amphénone B (inhibiteur corticosurrénalien) entraîne une hypertrophie marquée de l'interrénal chez Gasterosteus aculeatus et Betta splendens; simultanément, un index mitotique est établi après examen de 1.500 noyaux. Le diamètre nucléaire est mesuré sur 100 ou 200 noyaux, les valeurs obtenues étant présentées sous forme d'un histogramme, à classe unique pour les témoins. Selon les animaux, le traitement par l'Amphénone provoque un déplacement de cette classe de noyaux vers des valeurs plus élevées et l'apparition d'un ou peutêtre deux pics supplémentaires. L'ensemble des données quantitatives indique donc une forte stimulation confirmée par l'hypertrophie nucléolaire.

Chez l'Anguille, nous n'avous pas présenté les résultats obtenus sous forme d'histogrammes, mais il ne semble pas qu'un traitement stimulant déclenche l'apparition d'une seconde classe de noyaux de diamètre supérieur.

Chez Fundulus beteroclitus, PICKFORD (1953) préconise la mesure du nombre de couches cellulaires de l'interrénal limitant la veine cardinale, comme critère d'activité. Bien que moins précis que les tests précédents, celui-ci a l'avantage d'une grande simplicité et met en évidence une certaine atrophie glandulaire après hypophysectomie et une hypertrophie après injection d'ACTH. Toutefois, s'il est applicable aux inter- 
Tableau 3

Effets de divers traitements expérimentaux sur l'interrénal de l'Anguille

\begin{tabular}{|c|c|c|c|}
\hline Traitement & $\begin{array}{l}\text { Nombre } \\
\text { d'animaux }\end{array}$ & $\begin{array}{l}\text { Diamètres } \\
\text { nucléaires } \\
\text { en } \mu\end{array}$ & $\begin{array}{c}\text { Densités } \\
\text { nucléaires" }\end{array}$ \\
\hline Témoins non injectés & 6 & $4,61 \pm 0,01$ & $18,46 \pm 0,54$ \\
\hline Témoins injectés de $\mathrm{NaCl}$ & 15 & $4,67 \pm 0,04$ & $18,33 \pm 0,87$ \\
\hline Témoins + solvant de réserpine & 5 & $4,85 \pm 0,12$ & $16,86 \pm 0,61$ \\
\hline Métopirone & 33 & $5,45 \pm 0,08$ & $11,40 \pm 0,34$ \\
\hline Aldactone tous les 2 jours & 14 & $5,43 \pm 0,08$ & $9,25 \pm 0,30$ \\
\hline Aldactone quotidiennement & 12 & $5,60 \pm 0,04$ & $9,05 \pm 0,30$ \\
\hline Réserpine 2 à 9 jours & 6 & $5,19 \pm 0,09$ & $13,50 \pm 0,91$ \\
\hline Réserpine 2 à 25 jours & 16 & $4,93 \pm 0,06$ & $13,67 \pm 0,65$ \\
\hline Eau désionisée 7 à 15 jours & 12 & $4,63 \pm 0,06$ & $14,85 \pm 0,39$ \\
\hline Eau de mer 3 à 17 jours & 8 & $4,60 \pm 0,11$ & $16,80 \pm 0,70$ \\
\hline Cortisol 6 à 18 jours & 10 & $4,23 \pm 0,04$ & $26,30 \pm 0,74$ \\
\hline Hypophysectomie 15 à 30 jours & 7 & $3,84 \pm 0,09$ & $25,23 \pm 0,77$ \\
\hline Témoins & 7 & & $19,28 \pm 0,46$ \\
\hline Thy roxine & 6 & & $16,90 \pm 0,73$ \\
\hline Radiothyroïdectomie & 7 & & $25,75 \pm 0,81$ \\
\hline
\end{tabular}

rénaux de Fundulus, du Cyprin ou de Poecilia (Ball et al. 1965) dont les cellules forment un manchon concentrique à la veine logé dans le tissu lymphoïde du rein céphalique, il est moins adapté à la structure lobulaire de l'interrénal de l'Anguille et ne convient pas du tout pour les Salmonidés dont l'interrénal se développe en îlots de taille extrêmement variable au long des veines cardinales et de leurs moindres ramifications. De plus, cette mesure peut être faussée si la section n'est pas dans un plan perpendiculaire à celui de la veine, et si elle coupe tangentiellement les îlots glandulaires.

En résumé, ces divers procédés ont un but unique: traduire quantitativement les modifications histologiques de l'interrénal en réponse à des situations expérimentales variées. Malgré leurs imperfections, ils permettent de préciser si une réaction est statistiquement significative ou non et de renoncer aux vagues descriptions d'antan, mentionnant une «légère» hypertrophie ou une «discrète» atrophie, diagnostics peu compromettants et d'autant plus délicats à énoncer qu'il s'agit d'un tissu diffus, capricieusement réparti dans les veines cardinales et le rein lymphoïde.

Mais ces procédés ont un inconvénient commun, celui d'exiger un long travail ingrat, fatigant, qui incite maintenant les jeunes chercheurs à se détourner des études histophysiologiques pour aborder les techniques biochimiques qui apparemment leur promettent des résultats rapides et précis. 


\section{CORPUSCULES DE STANNIUS}

Si la fonction exacte des corpuscules de Stannius demeure encore mystérieuse, leur participation au contrôle des processus osmorégulateurs semble de plus en plus évidente (Fontaine 1964, Leloup-Hatey 1964b et c, Chester Jones et al. 1965) chez l'Anguille. Les corpuscules réagissent à divers traitements par des modifications assez complexes et difficiles à décrire, en particulier, lors d'une stimulation, une architecture

Tableau 4

Variations du volume nucléaire dans les corpuscules de Stannius de l'Anguille

\begin{tabular}{|lccc|}
\hline Traitement & $\begin{array}{c}\text { Nombre } \\
\text { d'animaux }\end{array}$ & $\begin{array}{c}\text { Volume } \\
\text { nucléaire } \\
\text { en } \mu^{3}\end{array}$ & $\begin{array}{c}\text { Signifi- } \\
\text { cation }\end{array}$ \\
\hline Témoins injectés de NaCl, mâles & 15 & $50,5 \pm 7,55$ & \\
Mâles traités par l'aldactone & 16 & $89,7 \pm 13,10$ & $\mathrm{P}<0,005$ \\
Témoins femelles & 2 & $35,2 \pm 5$ & \\
Témoins opérés femelles & 3 & $47,6 \pm 3,90$ & $\mathrm{P}<0,01$ \\
Hypophysectomisées depuis 8 à 14 mois & 4 & $74,9 \pm 3,90$ & $\mathrm{P}<0,001$ \\
Témoins femelles & 4 & $54,1 \pm 1,44$ & $105,1 \pm 4,80$ \\
Hypophysectomisées depuis 16 mois & 6 & $105,1 \pm 4$ & \\
\hline
\end{tabular}

cordonnale se substitue progressivement à une structure folliculaire. La mesure du diamètre nucléaire montre effectivement que l'administration d'aldactone (OlIVEREAU 1963d) ou lhypophysectomie (Olivereau \& Fontarne 1965) provoque, après des délais variables, une hypertrophie très nette du noyau, associée à une hyperplasie et à une richesse du cytoplasme en acide ribonucléique. Quelques unes de ces valeurs sont indiquées dans le tableau 4.

La maturation sexuelle du mâle, qu'elle soit provoquée par l'injection de prolans en eau de mer, ou par celle d'hormone thyréotrope en eau douce, s'accompagne aussi d'une hyperactivité des corpuscules de Stannius (Olivereau 1961b). Dans ce dernier cas, la planimétrie permet encore d'évaluer l'hypertrophie importante de ces organes. Le développement des structures ergastoplasmiques et une multiplication mitotique viennent confirmer le diagnostic d'hyperactivité.

\section{PANCREAS ENDOCRINE}

Le pancréas endocrine ou tissu langerhansien est disséminé en nodules assez gros, formant parfois quelques amas plus volumineux ou corps de Brockmann. Son volume peut aussi être évalué par planimétrie. C'est ainsi que le Père Palayer (1964) a mis en évidence une relation entre le volume total des îlots de Langerhans et la rhéonégativité des Anguilles au stade civelle.

Ce tissu est souvent composé de 3 ou 4 types de cellules dont les proportions varient selon l'état physiologique du poisson ou le traitement expérimental. La composition de ces populations cellulaires a été établie à la suite de numérations portant 
sur les deux îlots principaux, splénique et pylorique chez Cottus scorpius (FALKMER 1961).

\section{RÉSUME}

1. Nous avons exposé brièvement plusieurs techniques susceptibles d'associer quelques données quantitatives aux modifications qualitatives généralement décrites par les histophysiologistes dans les glandes endocrines des Téléostéens. Il est bien évident que ces deux aspects, qualitatifs et quantitatifs, ne doivent pas être considérés séparément, sinon leur interprétation risque d'être faussée.

2. L'activité de l'hypophyse peut être précisée par la caryométrie, mais l'étude des modifications cytologiques et histochimiques des divers types cellulaires de la pars distalis garde jusqu'ici la priorité sur les mesures quantitatives.

3. L'activité de la glande thyrö̈de, non individualisée, est surtout évaluée par la mesure de la hauteur épithéliale et par autohistoradiographie.

4. L'interrénal, diffus dans le rein céphalique et dans la paroì des veines cardinales, présente des variations volumétriques mesurables par planimétrie. Le diamètre nucléaire et le nombre de noyaux répartis sur une surface constante, sont deux indices traduisant fidèlement l'activité de l'interrénal et ses modifications en réponse à des traitements expérimentaux divers.

5. La planimétrie a été également appliquée à l'étude des corpuscules de Stannius et du pancréas, ainsi que la caryométrie. Toutes les données quantitatives sont ensuite soumises à un calcul statistique rigoureux permettant de vérifier la signification des résultats.

6. Ces méthodes ne sont pas à l'abri de toute critique, et il faut parfois en utiliser plusieurs pour avoir un tableau plus fidèle de l'activité glandulaire dans ces organes diffus. Nous avons montré quelques exemples expérimentaux dont les résultats ont bénéficié de l'application de ces techniques. Celles-ci, malgré leurs imperfections, permettent cependant l'introduction de données quantitatives qu'il est souhaitable de voir confronter avec les descriptions histologiques plus souvent subjectives.

\section{LITTERATURE CITÉE}

Ball, J. N., Olivereau, M., Slicher, A. M. \& Kallman, K. D., 1965. Functional capacity of ectopic pituitary transplants in a teleost fish, Poecilia formosa, with a comparative discussion on the transplanted pituitary. Phil. Trans. R. Soc. (Ser. B) 249, 69-99.

Benort, J. \& DA LAGE, C., 1963. Cytologie de l'Adénohypophyse. Colloques int. Cent. natn. Rech. scient. Paris 128, 1-434.

Chester Jones, I., Henderson, I. W. \& Butler, D. G., 1965. Water and electrolyte flux in the European eel (Anguilla angwilla L.). Archs Anat. microsc. Morph. exp. 54, 53-69.

- - \& Moscex, W., 1964. Methods for the adrenalectomy of the European eel (Anguilla anguilla L.). J. Endocr. 30, 155-156.

FAlKMER, S., 1961. Experimental diabetes research in fish. On the morphology and physiology of the endocrine pancreatic tissue of the marine teleost Cottus scorpins with special reference to the role of glutathione in the mechanism of alloxan diabetes using a modified nitroprussid method. Acta endocr., Copenh. 37 (Suppl. 59) 1-122. 
Folrenius, E., 1963. Ultrastructure des types cellulaires de 1'hypophyse de quelques poissons téléostéens. Archs Anat. microsc. Morph. exp. 52, 429-468.

Fontaine, M., 1961. Action d'un extrait antéhypophysaire thyréostimulant sur la maturation des ovaires de l'Anguille femelle (Anguilla anguilla L.). C. r. bebd. Séanc. Acad. Sci, Paris 252, 3365-3367.

- Callamand, O. \& Vibert, R., 1950. La physiologie du Saumon. Annls Stn cent. Hydrobiol. appl. 3, 15-26.

- Leloup, J. \& Oltvereau, M., 1952. La fonction thyroïdienne du jeune Saumon, Salmo salar L. (PARR et SMOLT) et son intervention possible dans la migration d'avalaison. Archs Sci. physiol. 6, 83-104.

- \& Olivereau, M., 1957. Interrénal antérieur et smoltification chez Salmo salar L. J. Pbysiol. Paris $9,174-176$.

- - 1959. Interrénal antérieur et smoltification chez Salmo salar L. Etude volumétrique. Bull. Soc. Zool. Fr. 84, 161-162.

Gracomini, E., 1908a. Sulla disposizione del sistema interrenale e del sistema feocromo nelle Anguille adulte, nelle cieche e nei Leptocefali. Rc. Sess. Accad.Sci. Ist. Bologna 12, 172-176.

- 1908b. Il sistema interrenale e il sistema cromaffine (sistema feocromo) nelle anguille adulte, nelle cieche e nei leptocefali. Memorie R. Accad. Sci. Ist. Bologna (Ser. 6) 6, 407-441.

La Roche, G., Johnson, C. L. \& Woodalt, A. N., 1965. Communication personnelle.

Leloup-Hatey, J., 1964a. Fonctionnement de l'interrénal antérieur de deux téléostéens, le Saumon atlantique et l'Anguille européenne. Annls Inst. océanogr., Monaco 43, 221-338.

- 1964b. Modifications de l'équilibre minéral de l'Anguille (Anguilla anguilla L.) consécutives à l'ablation des corpuscules de Stannius. C. r. Séanc. Soc. Biol. 158, 711-715.

- 1964c. Influence d'un apport sodique et de l'aldostérone sur le déséquilibre minéral consécutif à l'ablation des corpuscules de Stannius chez l'Anguille (Angwilla angrilla L.). C. $r$. Séanc. Soc. Biol. 158, 991-994.

Olivereau, M., 1954. Hypophyse et glande thyroïde chez les poissons. Etude histophysiologique de quelques corrélations endocriniennes en particulier chez Salmo salar L. Annls Inst. océanogr., Monaco 29, 95-296.

- 1957. Radiothyroïdectomie chez l'Anguille (Anguilla anguilla L.). Archs Anat. microsc. Morph. exp. 46, 39-59.

- 1960a. Action de la thyroxine sur l'hypothalamus, l'hypophyse et la glande thyroïde de 1'Anguille. Anat. Anz. 109, 468-478.

- 1960b. Etude anatomique et histologique de la glande thyroïde d'Uegitglanis zammaranoi Gtanferrari, poisson aveugle et cavernicole. Comparaison avec un Clariidae voisin, Clarias butbupogon A. Dum. Annls Soc. r. Zool. Belg. 90, 99-116.

- 1960c. Quelques aspects anatomiques et physiologiques de la glande thyroïde des poissons. Annls Soc, r. zool. Belg. 90, 83-98.

- 1960d. Etude volumétrique de l'interrénal antérieur au cours de la smoltification de Salmo salar L. Acta endocr., Copenh. 33, 142-156.

- 1961a. Maturation sexuelle de l'Anguille mâle en eau douce. C. r. bebd. Séanc. Acad. Sci., Paris 252, 3660-3662.

- 1961b. Corpuscules de Stannius et reproduction chez l'Anguille mâle. C. r. hebd. Séanc. Acad. Sci., Paris 253, 541-543.

- 1962. Cytologie de l'hypophyse du Cyprin (Carassius auratus L.). C. r. bebd. Séanc. Acad. Sci., Paris 255, 2007-2009.

- 1963a. Effets de la radiothyrö̈dectomie sur l'hypophyse de l'Anguille. Discussion sur la 〈pars distalis' des Téléostéens. Gen. comp. Endocr. 3, 312-332.

- 1963b. Cytophysiologie du lobe distal de l'hypophyse des Agnathes et des Poissons à l'exclusion de celle concernant la fonction gonadotrope. Colloques int. Cent, natn. Rech. scient. Paris 128, 315-330.

- 1963c. Action de la réserpine sur l'hypophyse, l'interrénal et les cellules chromaffines de l’Anguille, Anguilla anguilla L. C. r. Séanc. Soc. Biol. 157, 1357-1360.

- 1963d. Action de l'aldactone sur les corpuscules de Stannius de l'Anguille. C. r, bebd. Séanc. Acad. Sci., Paris 257, 4035-4038. 
- 1964. L'hématoxyline au plomb permet-elle l'identification des cellules corticotropes de I'hypophyse des Téléostéens? Z. Zellforsch. mikrosk. Anat. 63, 496-505.

- 1965. Action de la métopirone chez l'Anguille normale et hypophysectomisée, en particulier sur le système hypophyso-corticosurrénalien. Gen. comp. Endocr. 5, 109-128.

- 1966. Action de la prolactine chez l'Anguille intacte et hypophysectomisée. I Système hypophyso-thyrö̈dien et pigmentation. Gen. comp. Endocr. 6, 130-143.

- \& Chartier, M. M., 1965. Aldactone et osmorégulation chez l'Anguille. C. r. Séanc. Soc. Biol. 159, 1498-1503.

- \& Fontarne, M., 1965. Effet de l'hypophysectomie sur les corpuscules de Stannius de l'Anguille. C. r. bebd. Séanc. Acad. Scí, Paris 261, 2003-2008.

- \& Fromentrn, H., 1954. Influence de l'hypophysectomie sur l'histologie de l'interrénal antérieur de l'Anguille (Anguilla angwilla L.). Annls Endocr. 15, 805-826.

- \& LA Roche, G., 1965. Effects of low iodine intake or destructive doses of ${ }^{131} \mathrm{I}$ on pituitary histochemistry of young chinook salmon (Oncorbynchus tshareytscha Walbaum). Am. Zool. $5,234$.

- - \&x Woodall, A. N., 1964. Modifications cytologiques de l'hypophyse de la Truite à la suite d'une carence en iode et d'une radiothyroïdectomie. Annls Endocr. 25, 481-490.

OVERBEEKE, A. P. VAN, 1960. Histological studies on the interrenal and the phaeochromic tissue in teleostei. Amsterdam, Thèse de l'Univ. Libre, $102 \mathrm{pp}$.

PALAYER, P., 1964. Corrélations entre le rhéotropisme et morphogénèse d'une part et structure histologique du pancréas endocrine d'autre part chez la Civelle (Anguilla anguilla L.). Annls Endocr. 25, 619-624.

Pickford, G. E., 1953. A study of the hypophysectomized male killifish, Fundulus heteroclitus (Linn.). Bull. Bingham oceanogr. Coll. 14, 5-41.

Schreibman, M. P., 1964. Studies on the pituitary gland of Xiphophorus maculatus (the platyfish). Zoologica, N.Y. 49, 217-243.

STANWORTH, P., 1953. A study of reproduction in the three spined stidkleback, Gasterostews aculeatus L., with some reference to endocrine control. Sheffield, Ph. D. thesis, 213 pp.

\section{Discussion suivant le discours de OLIVEREAU}

SCHARF: (1) Was ist Metopiron und wer stellt es her? (2) Wieviel Messungen (etwa Zellkerne) haben Sie pro Organ durchgeführt? (3) Wie können Sie garantieren, daß der wesentliche Anteil der disseminierten Thyreocyten erfaßt wird? Das geht doch nur bei langdauernder Blockade mit ${ }^{131} \mathrm{~J}$. Ich bedaure, daß Ihre schönen Arbeiten häufig mißverstanden werden. Sie könnten es sich und Ihren Lesern leichter machen, wenn Sie sich bei der Nomenklatur der Hypophysenvorderlappenzellen an die international angenommenen Vereinbarungen hielten. Der Nomenklaturvorschlag stellt dem Autor frei, entweder die griechischen Buchstaben oder die lateinischen zu verwenden, nicht aber die färbetechnischen Namen.

OlrvereaU: (1) La métopirone ou SU 4885 est fabriquée par la maison CIBA. (2) Le nombre de noyaux mesurés est de 200 en général. Pour les numérations sur une aire constante de $1600 \mu^{2}$, le nombre de noyaux comptés varie de 400 à 750 suivant leur densité. (3) La nomenclature utilisée pour les cellules pituitaires est celle adoptée lors du Colloque international, organisé par le Centre de la Recherche Scientifique (CNRS) à Paris, en Septembre 1963 (Cytologie de l'adénohypophyse. Ed. par J. Benoit \& C. Da Lage. Colloques int. Cent. natn. Rech. scient. 128, 1-434.) au cours duquel 22 rapports furent présentés par les spécialistes de cette question. Cette nomenclature a surtout été basée sur les résultats des recherches expérimentales du Professeur HERLANT et de ses divers collaborateurs européens travaillant sur les différentes classes de Vertébrés. Elle a également été utilisée lors de la Table Ronde sur l'Hypophyse, au 4ième Symposium International d'Endocrinologie Comparée (Paris, 1964). La terminologie dont vous parlez a été proposée par Ezrin au Canada, mais elle est uniquement basée sur l'étude de l'hypophyse humaine, surtout pathologique, ce qui la rend difficilement applicable à tous les Vertébrés. La confusion que vous éroquez au sujet des cellules $\beta$ et $\delta$ vient du fait 
que les travaux de Romers ne furent pas correctement interprétés par les premiers auteurs anglo-saxons, mais toute la clarté sur cette discussion a été faite, en particulier par HERLANT, Halmi, Purves et Racadot, à la suite du Colloque International. Et pour éviter de prolonger cette confusion, le voeu a été émis que l'on utilise de plus en plus une nomenclature physiologique, en omettant les lettres grecques, et que l'on parle de cellules thyréotropes au lieu de cellules $\beta$ ou $\delta$. Chez l'Anguille, la terminologie n'est pas basée sur de simples réactions tinctoriales, mais sur les résultats d'une étude expérimentale portant sur plus de 800 animaux, à l'aide de techniques cytologiques et histochimiques. Les cellules $\varepsilon$ corticotropes ont été identifées chez divers Vertébrés, en particulier chez l'Anguille, après traitement par la métopirone, l'aldactone, la réserpine, l'o, $\mathrm{p}$ 'DDD, le cortisol ...., et les cellules thyréotropes après radiothyroïdectomie ou administration de thyroxine, les éléments gonadotropes par l'étude en fonction du développement génital expérimentalement induit.

SCHARF: Ich halte 200 Messungen pro Organ für zu wenig. Die Befunde sind nicht reproduzierbar, M. PALKovits (Z. mikrosk, anat. Forsch. 67, 343, 1961; Endocrinology 45, 227, 1963) hat viel Arbeitskraft investiert. Er konnte zeigen, daß weniger als 1000 Zell- oder Kernmessungen pro Organ zu wenig sind; oft werden 5000 gebraucht. Ich verweise auf die Nomina histologica!

Olfvereau: Je connais ce travail d'un intérêt capital, mais je ne pense pas que le travail supplémentaire considérable pour mesurer plus de 200 noyaux accroisse beaucoup la précision de nos données, toutes les valeurs étant obtenues par un procédé identique. De plus, les noyaux de l'interrénal ont généralement une taille très homogène, et BASU et al. (1965) se contentent de mesurer seulement 100 noyaux dans ce même tissu. Avec le nombre d'animaux traités à chaque expérience, l'erreur standard des diamètres nucléaires est inférieure à $0,1 \mu$. Dans les expériences d'inhibition corticosurrénalienne (par exemple après métopirone ou aldactone), les differences entre animaux témoins et traités sont tellement importantes (volume nucléaire passant de 50 à 80 ou $90 \mu^{3}$ ) qu'elles ne semblent pas justifier des mesures portant sur 1000 ou 5000 noyaux pour être significatives. Il en est de même pour les densités nucléaires qui varient du simple au double selon les traitements. Je crois qu'il ne faut pas demander à l'histophysiologie plus qu'elle ne peut nous donner, elle reste surtout qualitative et si l'on souhaite accroître la précision des résultats, il faut savoir se tourner vers la biochimie.

Marmasse: Which statistical evaluation of your data has been performed?

Olivereav: Les décimales indiquées dans les tableaux des valeurs correspondent simplement à la moyenne des valeurs individuelles, les divers groupes expérimentaux comprenant de 6 à 33 individus. Les erreurs standards se trouvent indiquées dans les tableaux figurant dans le texte. Les calculs statistiques effectués sur toutes les valeurs citées montrent que la plupart des différences observées après les divers traitements expérimentaux sont significatives, la valeur de $\mathrm{P}$ indiquant le degré de cette signification.

MILLER: (1) Were your quantitative measurements of the diameter of the cells and nuclei made on tissues subjected to chemical fixation and paraffine embedding? (2) Since these procedures may produce great and variable shrinkage or swelling of cells and organelles, would it not be well to check these measurements on tissues prepared by freeze-drying or freeze-drying substitution?

OLIVEREAu: Les valeurs indiquées sont obtenues sur du matériel fixé. Il faut tenir compte d'une certaine rétraction liée à la fixation et aux divers processus histologiques, en particulier à la déshydratation. Nous comparons des coupes toujours obtenues dans des conditions identiques, de même épaisseur, et il semble que l'utilisation de l'Autotechnicon permette de standardiser les conditions de travail et réduire au minimum les variations au cours du traitement des tissus, éliminant ainsi un facteur personnel. Nous n'avons pas utilisé la technique du freezingdrying qui permettrait de préciser les effets liés aux processus de la préparation des coupes.

PRECHT: Auf dem 1. Internationalen Symposion über Probleme der quantitativen Biologie des Stoffwechsels habe ich versucht, die Bedeutung der Schilddrüse für die Resistenzadaptation theoretisch zu behandeln. Wir würden uns freuen, wenn Mme Olivereau durch dieses Symposion für diese Probleme interessiert werden könnte. 